\title{
Influence of bone morphogenetic protein on articular cartilage regeneration following periosteal grafting
}

\author{
Yimin Zhang, Xin Jiang, Yongzhi Guo \\ Orthopedic Department, Weifang People's Hospital, Weifang, China
}

Email:ymzh69@hotmail.com

Received 4 December 2012; revised 11 January 2013; accepted 20 January 2013

\begin{abstract}
Background: Autologous periosteal grafting is used as treatment for articular cartilage defect. Objective: To study the effect of bone morphogenetic protein (BMP) on articular cartilage regeneration following periosteal grafting. Methods: 16 healthy 15-week-old New Zealand white rabbits of both sexes (32 knees) were randomly divided into experimental group (group A) and control group (group B). A $4.0 \mathrm{~mm}$ diameter full-thickness articular cartilage defect was created in the femoral intercondylar fossa in all rabbits. Following this, a $4.0 \mathrm{~mm}$ diameter section of the periosteum was harvested from the anteromedial part of the upper tibial bone. In group A (eight rabbits, 16 knees), the cartilage defect was covered with periosteum, into which $20 \mu \mathrm{g}$ BMP and $20 \%$ Pluronic were injected. In group $B$ (eight rabbits, 16 knees), the cartilage defect was covered with periosteum, into which the same dosage of $0.9 \%$ NS (Normal saline) and $20 \%$ Pluronic were injected. All rabbits were sacrificed at 4,8 , and 12 weeks postoperatively, the cartilage defect areas were examined macroscopically and microscopically, and the morphology of the chondrocytes and collagen fibers were examined by scanning electron microscopy. Results: The filling of the defects with regenerated tissue was observed in both the group. The most notable improvement was that the cartilage regeneration in group A was obviously superior to that in group $B$, with the total histological score in group A significantly higher. Conclusion: BMP is an effective factor that could promote regeneration of articular cartilage and lead to successful cartilaginous resurfacing following periosteal grafting.
\end{abstract}

Keywords: Bone Morphogenetic Protein (BMP); Articular; Cartilage; Regeneration; Periosteal Grafting

\section{INTRODUCTION}

The repair of the articular cartilage is one of the most widely researched areas in modern medicine. Injury and defects of articular cartilage can lead to traumatic arthritis, because of limitations in self-regeneration and selfrepair of cartilage. It has been shown [1] that a considerable number of undifferentiated mesenchymal stem cells (MSCs) exist extensively in the periosteum, which have the potential for multipotent differentiation and great capacity for self-renewal, similar to hemopoietic stem cells (HBSCs) in the blood.

Periosteum as a type of mesenchymal tissue, can be transplanted into defect areas in articular cartilage for repair and regeneration, because of its three primary requirements for tissue engineering [2] (i.e. provides matrix, source of stem cells and growth factors). It has been proved that this procedure in the long term showed improved results [3].

Bone morphogenetic proteins (BMPs) are family of growth and differentiation factors, belonging to the transforming growth factor $\beta$ superfamily [1,4]. In 1965, the capability of bone induction of decalcified bone matrix was first reported by Urist [5]. It is well known that BMPs are pleiotropic morphogens, and they induce new cartilage and bone formation in ectopic sites by a developmental sequence that mimics limb development and morphogenesis [6], and the application of BMPs can have a positive stimulatory function on repair and regeneration [4].

In this experiment, we performed a control study to verify the effects of BMP on articular cartilage regeneration following periosteal grafting.

\section{MATERIALS AND METHODS}

\subsection{Preparation of the Pluronic Gel}

20 mg Pluronic F-127 (Sigma Corp.) was slowly added to a clean glass bottle containing $100 \mathrm{ml}$ distilled water. The glass bottle with the mixture of Pluronic gel and distilled water was oscillated under $4^{\circ} \mathrm{C}$ for $4 \mathrm{~h}$, until the flocculent particles were completely dissolved. The glass bottle containing the mixture was then irradiated under Cobalt60 for $3000 \mathrm{cGy}$ until all bacteria were eradicated, which was confirmed by bacterial culture. 


\subsection{Experimental Animals}

16 healthy 15 -week-old New Zealand white rabbits, weighing $2.5-3.0 \mathrm{~kg}$, were used in this study. All fore knees ( 32 in total) were used for analysis.

\subsection{Preparation of Models}

A $20 \mathrm{~g} / \mathrm{L}$ solution of barbital sodium was injected through the auricular vein at a dosage of $200 \mathrm{mg} / \mathrm{kg}$ for general anesthesia. After shaving and disinfecting the operating area, a medial parapatellar approach of a $5 \mathrm{~cm}$-long incision was used and the patella was dislocated laterally to expose the knee joint. A $4.0 \mathrm{~mm}$ diameter drill was then applied to create a full-thickness cartilage defect in the middle part of the femoral inter condylar fossa. Then $4.0 \mathrm{~mm}$ diameter of periosteum was excised from the anteromedial part of the upper tibial bone for free grafting.

\subsection{Experimentation}

The rabbits were randomly divided into experimental group (eight rabbits, 16 fore knees) and control group (eight rabbits, 16 fore knees) in accordance with sortition.

Experimental group (group A): $20 \mu \mathrm{g}$ BMP (Sigma corp.) was microinjected into the cartilage defect, and the defect area was sealed with $20 \%$ Pluronic gel. The periosteum obtained from the tibial bone was used as a graft for coverage with its cambium layer facing up to the knee joint. The edge of the graft was fixed by suture with the surrounding bone.

Control group (group B): $20 \mu \mathrm{g}$ 0.9\% NS (Normal saline) was microinjected into the cartilage defect, and the defect area was sealed with $20 \%$ Pluronic gel. The periosteum was also grafted similarly to the experimental group.

After finishing grafting, all debris was removed and the joint was closed by suturing. All rabbits were raised freely in a cage without external fixation of the affected limbs. 4, 8 and 12 weeks after surgery, the rabbits were sacrificed by anesthesia, the operated knee joints were excised, carefully cleaned of all neighboring tissues, subjected to macroscopic, histological and ultrastructural study.

\subsection{Statistical Analysis}

Software SPSS11.0 version was used for statistical analysis by the first author with the experimental data. A $\mathrm{P}$ value of $<0.05$ denotes a statistically significant difference.

\section{RESULTS}

\subsection{Inclusion of the Experimental Animals}

All 16 rabbits were included in the analysis.

\subsection{Macroscopic Study}

In all rabbits, there was a small volume of yellowish, lucid synovial fluid in the knee joints, and there was no contracture of the articular capsule, amotio of repair tissue, or swelling and hyperemia of the synovium. The surrounding cartilage kept well.

Four weeks after surgery, in the experimental group, the cartilage defect area was replaced by semitransparent, smooth, pink and slightly swollen cartilage-like tissue, which formed a clear boundary from the surrounding cartilage. In the control group, the cartilage defect was covered by white, opaque, soft periosteum, the basilar surface of which was cardinal red, and a clear separation from the surrounding tissue. Eight weeks later, in the experimental group, the repair tissue was smooth, pink, flexible, and the boundary with the surrounding cartilage was obscure. In the control group, the periosteum remained white, soft, rough with no flexibility, and a clear separation from the surrounding surface. At 12 weeks, in the experimental group, the color and texture of the repair tissue grafted on the cartilage defect was similar to that of normal cartilage and the boundary with the surrounding cartilage was disappearing. The surface of the repair tissue was almost at the same level as the surrounding tissue. Compared with the experimental group, the repair tissue in the control group was mostly smooth, but had only soft texture and no flexibility. However, the boundary between the repair tissue and surrounding surface had become obscure.

Total mean scores of the two groups according to the macroscopic scoring system described by CarranzaBencano et al. [7] are shown in Table 1. Analysis of variance was performed using Dunnett's test, compared with the control group. Although the scores were higher in group A (experimental group), there was no statisticcally significant difference between the two groups ( $p>$ $0.05)$.

\subsection{Histological Study under Microscopy}

4 weeks after surgery, in experimental group, the area of cartilage defect was filled with cells and matrix, with the cambium layer of periosteum increasing and differentiating. The basilar part of the cambium layer began to form a fibrous connection with the surrounding tissue

Table 1. Macroscopic scores of each group at different time points according to Carranza-Bencano scoring system [7].

\begin{tabular}{cccc}
\hline Group & Weeks 4 & Weeks 8 & Weeks 12 \\
\hline Control & 8 & 10 & 12 \\
Experimental & 10 & 13 & 14 \\
\hline $\mathrm{P}>0.05$, vs control group. & & &
\end{tabular}


(Figure 1(a)). In the control group, the cambium layer increased slightly, there was very little differentiation, and there was no binding with the surrounding tissue (Figure 2(a)). Eight weeks later, in the experimental group, the characteristic morphology of fibrous cartilage was visible; the periosteum graft had thickened and contained round or ovate cells. The cell density exceeded that of normal cartilage with an obvious cartilage capsule. The repair tissue was slightly higher than its surrounding surface and some hyaline cartilage was visible (Figure 1(b)). In the control group, no periosteum thickening occurred, few round or ovate cells were visible and no hyaline cartilage formation was detected. A very small amount of fibrous cartilage could be seen and there was a clear boundary with the surrounding tissue (Figure 2(b)). Twelve weeks postoperatively, the defects in the experimental group healed well and there was a reduced cell density and uniform matrix. The cell shape and alignment were similar to normal cartilage. Among the experimental knees, eight knees showed excellent healing with the surrounding cartilage; most of the cartilage regenerated from the periosteum was hyaline in six knees, and there was incomplete binding with the surrounding tissue. Vertical cartilago styliformis (typical articular cartilage) was seen in two knees (Figure 1(c)). In the control group, the surface of the grafted tissue was rough, the formation of fibrous tissue was obvious, and only a small amount of fibrous cartilage could be observed. No hyaline cartilage or reintegration with the surrounding tissue could be found (Figure 2(c)).

The scores of microscopic histology according to Wakitani score system [8] are shown in Table 2. Analysis of variance was performed using Dunnett's test, compared with the control group. The total score on the histological grading were significantly better in group A (experimental group) than group $\mathrm{B}$ (control group), $\mathrm{P}<$ 0.05 .

\subsection{Ultrastructural Study under SEM}

In the experimental group, 4 weeks after surgery, there were round cells with orbicular-ovate nuclei and irregular processes next to fibrous tissue in the repair tissue (Figure 3(a)). After another 8 weeks, the introcession of nucleus, abundant rough endoplasmic reticulum (RER) and glycogenosome in endochylema, with microfilament (MF) and sap cavity, and irregular collagen fibrils were seen in the matrix (Figure 3(b)). Twelve weeks postsurgery, in the experimental group, clear nuclear membranes, well-distributed chromoplasm, round cells with active proliferation, abundant rough endoplasmic reticulum (RER) with a distention of layers, small round mitochondria, developed Golgi apparatus, sap cavity, and lipid droplets in endochylema were observed. Furthermore, mature hyaline cartilage had formed and there was a productive secretion of cartilage matrix from the abundant cells. The intercellular spaces were linked to the cartilage matrix with a fluffy prominence on the surface

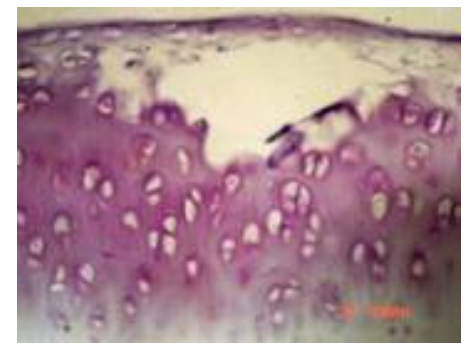

(a)

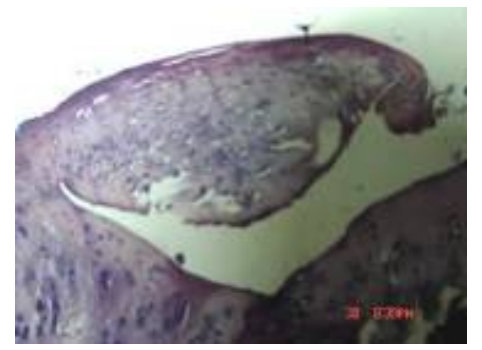

(b)

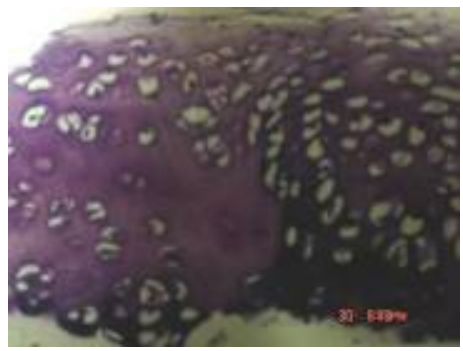

(c)

Figure 1. Histological study of the grafting periosteum in experimental group (Haematoxylin-eosin staining, $\times 100$ ). (a) 4 weeks; (b) 8 weeks; (c) 12 weeks.

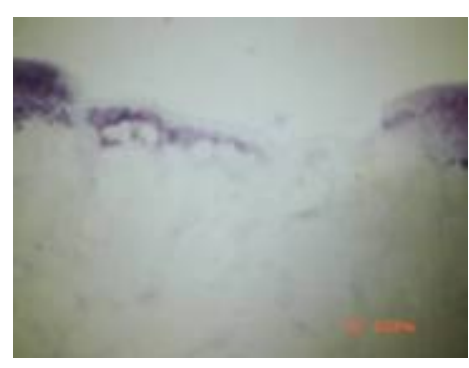

(a)

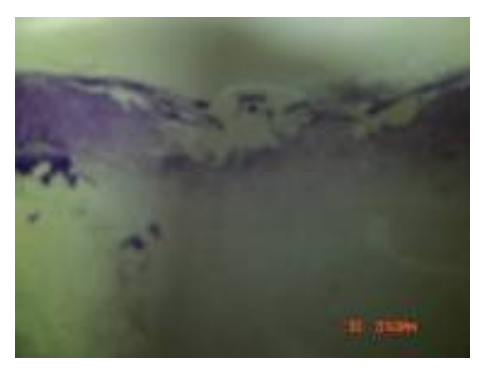

(b)

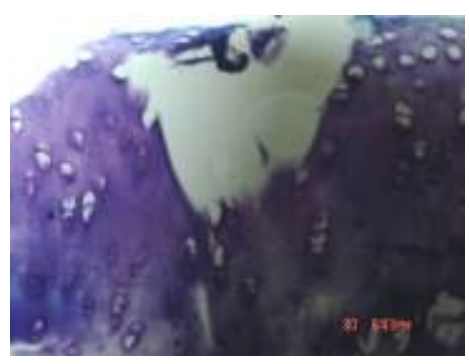

(c)

Figure 2. Histological study of the grafting periosteum in control group (Haematoxylin-eosin staining, $\times 100$ ). (a) 4 weeks; (b) 8 weeks; (c) 12 weeks. 
Table 2. Histological scores of each group at different time points according to Wakitani scoring system [8] $(\bar{x} \pm \mathrm{s}$, point).

\begin{tabular}{cccc}
\hline Group & Weeks 4 & Weeks 8 & Weeks 12 \\
\hline Control & $9.67 \pm 0.55$ & $8.64 \pm 0.86$ & $7.02 \pm 0.61$ \\
Experimental & $4.03 \pm 0.88$ & $3.41 \pm 0.27$ & $3.06 \pm 0.48$ \\
\hline
\end{tabular}

$\mathrm{P}<0.05$, vs control group.

(Figure 4(c)). In the control group, a stratified alignment of fibrous tissue presented at 4 weeks (Figure 4(a)). At 8 and 12 weeks, most of the repair tissue was also in a stratified alignment of fibrous tissue, with only a little inactive fibrous cartilage (Figures 4(b) and (c)).

\section{DISCUSSION}

The periosteum consists of fibre and cambium layers. The cambium layer is strongly multipotent and has a large capacity for self-renewal, with copious mesenchymal stem cells (MSCs) $[1,9,10]$. The MSCs can quickly differentiate into cells of osteoblast lineage and gradually differentiate into cartilage and osteocytes when stimulated with specific factors $[11,12]$.

Basic research has revealed the good potential of periosteal grafting for cartilage regeneration $[9,10,13]$, and recent study has shown the process of cartilage regeneration following periosteal autograft [14]. Periosteal transplantation has become an effective approach to repair cartilage defects [15]. Its use in bioengineering applica- tions is practicable and there have been numerous studies reporting favorable results [16-19].

It has been well known that autogenic periosteum is an ideal material for cartilage repair, which can also be induced and enhanced by certain growth-differentiation factors, such as cartilage growth factor (CGF), transforming growth factor $\beta$ [20], fibroblast growth factor (bFGF) [21] and bone morphogenetic protein (BMP) $[16,22]$.

BMPs are family of growth and differentiation factors that are chemotactic and concentration-dependent, and that promote karyokinesis and induce differentiation. The functions of BMPs have been reported in many in vitro studies. In structure, BMP is a dimer formed by disulfide bonds, the bionomics of which are the induction of bone and cartilage regeneration, which can strongly promote alignment, differentiation and direction of bone and cartilage formation [13]. Furthermore, it has been shown that cartilage defect can be repaired following direct injection of BMP into the knee joints of rabbits without periosteal grafting [16]. Another studies on cartilage repair using growth-differentiation factors were also reported, such as recombinant human bFGF application following periosteal grafting [21] and pre-injection of TGF $\beta 1$ into the periosteum for grafting [20], both of which were effective for promoting the quality of regenerated cartilage. But to our knowledge, no previous study regarding the method of using BMP as an enhancing factor by the direct injection into the cartilage defect following periosteal grafting has been reported.

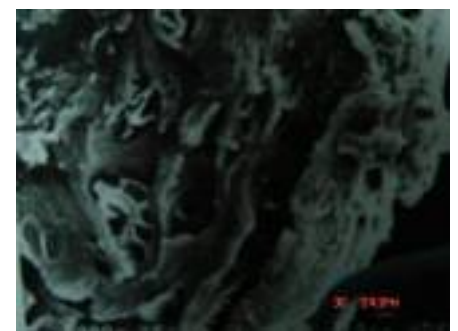

(a)

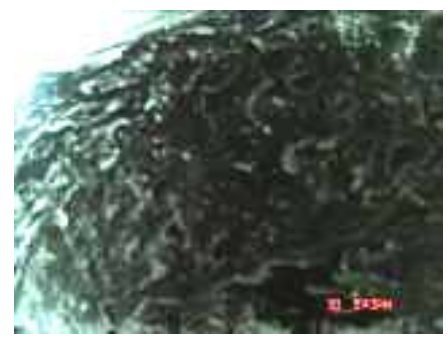

(b)

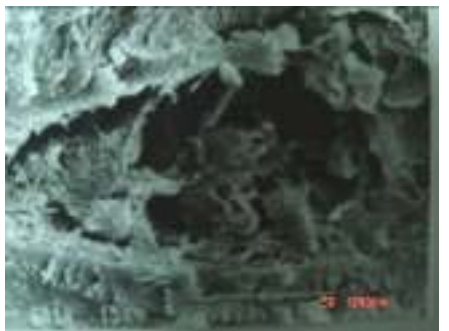

(c)

Figure 3. Ultramicrostructure of the grafting periosteum in experimental group under scanning electron microscopy (×7000); (a) 4 weeks; (b) 8 weeks; (c) 12 weeks.

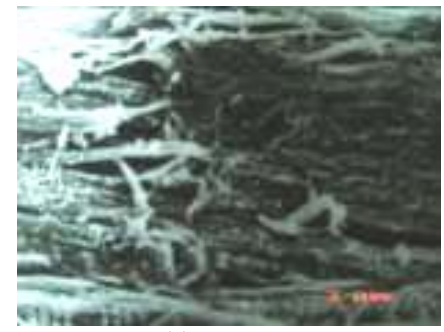

(a)

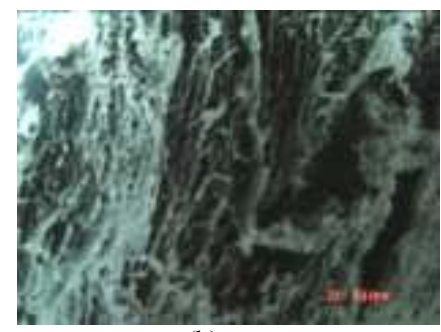

(b)

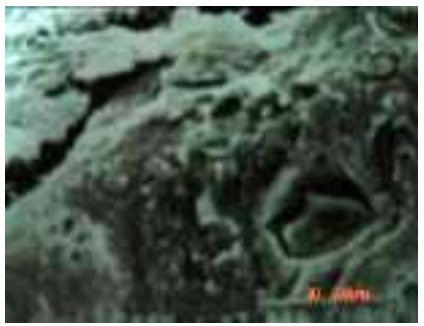

(c)

Figure 4. Ultramicrostructure of the grafting periosteum in control group under scanning electron microscopy (×7000); (a) 4 weeks; (b) 8 weeks; (c) 12 weeks. 
In this study, BMP was injected into the cartilaginous defect area following periosteal grafting to enhance the regeneration of new cartilage. Compared with the control group, BMP could significantly promote the process and efficacy of cartilage regeneration and repair.

As for the choose of Pluronic F-127 as the carrier, because it has the following advantages: 1) Satisfactory biocompatibility and biodegradability, with a degradation rate matching cartilage regeneration; 2) Suitability for injection because it undergoes liquefaction at $4^{\circ} \mathrm{C}$ and solidification at $37^{\circ} \mathrm{C}$; 3) It is non-antigenic and nonimmunogenic, and seldom reacts with other proteins and biomolecules [23].

But our follow-up period was short and the durability of the repair tissue was not given. Further investigations are needed.

\section{REFERENCES}

[1] Bruder, S.P., Jaiswal, N. and Haynesworth, S.E. (1997) Growth kinetics,self-renewal,and the osteogenic potential of purified human mesenchymal stem cells during extensive subcultivation and following cryopreservation. Journal of Cellular Biochemistry, 64, 278-294.

doi:10.1002/(SICI)1097-4644(199702)64:2<278::AID-JC B11>3.0.CO;2-F

[2] Langer, R. and Vacanti, J.P. (1993) Tissue engineering. Science, 260, 920-926. doi:10.1126/science.8493529

[3] O'Driscoll, S.W. and Fitzsimmons, J.S. (2001) The role of periosteum in cartilage repair. Clinical Orthopaedics and Related Research, 391, S190-S207. doi:10.1097/00003086-200110001-00019

[4] Lietman, S.A., Yanagishita, M., Sampath, T.K. and Reddi, A.H. (1997) Stimulation of proteoglycan synthesis in explants of porcine articular cartilage by $\gamma \mathrm{OP}-1$ (BMP7). The Journal of Bone \& Joint Surgery, 79, 1132-1137.

[5] Urist, M.R. (1965) Bone formation by autoinduction. Science, 150, 893-899. doi:10.1126/science.150.3698.893

[6] Reddi, A.H. (1998) Role of morphogenetic proteins in skeletal tissue engineering and regeneration. Nature Biotechnology, 16, 247-252. doi:10.1038/nbt0398-247

[7] Carranza-Bencano, A., García-Paino, L., Armas Padrón, J.R. and Cayuela Dominguez, A. (2000) Neochondrogenesis in repair of full-thickness articular cartilage defects using free autogenous periosteal grafts in the rabbit. A follow-up in six months. Osteoarthritis Cartilage, 8, 351-358. doi:10.1053/joca.1999.0309

[8] Wakitani, S., Goto, T., Pineda, S.J., Young, R.G., Mansour, J.M., Caplan, A.I. and Goldberg, V.M. (1994) Mesenchymal cell-based repair of large full-thickness defects of articular cartilage. The Journal of Bone \& Joint Surgery, 76, 592-597.

[9] Marion, N.W. and Mao, J.J. (2006) Mesenchymal stem cells and tissue engineering. Methods in Enzymology, 420, 339-361. doi:10.1016/S0076-6879(06)20016-8

[10] Oreffo, R.O., Cooper, C., Mason, C. and Clements, M.
(2005) Mesenchymal stem cells lineage, plasticity, and skeletal therapeutic potential. Stem Cell Reviews, 1, 169178. doi:10.1385/SCR:1:2:169

[11] Leo, A.J. and Grande, D.A. (2006) Mesenchymal stem cells in tissue engineering. Cells Tissues Organs, 183, 112-122. doi: $10.1159 / 000095985$

[12] Chen, F.H., Rousche, K.T. and Tuan, R.S. (2006) Technology Insight: adult stem cells in cartilage regeneration and tissue engineering. Nature Clinical Practice Rheumatology, 2, 373-382. doi:10.1038/ncprheum0216

[13] Luyten, F.P., Yu, Y.M., Yanagishita, M., Vukicevic, S., Hammonds, R.G. and Reddi, A.H. (1992) Natural bovine osteogenin and recombinant BMP-2B are equipment in the maintenance of proteoglycans in bovine articular cartilage explant cultures. Journal of Biological Chemistry, 267, 3685-3691.

[14] Sung, M.S., Jeong, C.H., Lim, Y.S., Yoo, W.J., Chung, S.K. and Jung, N.Y. (2011) Periosteal autograft for articular cartilage defects in dogs: MR imaging and ultrasonography of the repair process. Acta Radiologica, 52, 181-190. doi:10.1258/ar.2010.100087

[15] Messner, K. and Gillquist, J. (1996) Cartilage repair. A critical review. Acta Orthopaedica Scandinavica, 67, 523-529. doi:10.3109/17453679608996682

[16] Sellers, R.S., Zhang, R., Glasson, S.S., Kim, H.D., Peluso, D., D'Augusta, D.A., Beckwith, K. and Morris, E.A. (2000) Repair of articular defects one year after treatment with recombinant human bone morphogenetic protein2(rhBMP-2). The Journal of Bone \& Joint Surgery, 82, $151-160$

[17] Gallay, S.H., Miura, Y., Commisso, C.N., Fitzsimmons, J.S. and O'Driscoll, S.W. (1994) Relationship of donor site to chondrogenic potential of periosteum in vitro. Journal of Orthopaedic Research, 12, 515-525. doi:10.1002/jor.1100120408

[18] O’Driscoll, S.W., Recklies, A.D. and Poole, A.R. (1994) Chondrogenesis in periosteal explants. An organ culture model for in vitro study. The Journal of Bone \& Joint Surgery, 76A, 1042-1051.

[19] Slynarski, K., Deszczynski, J. and Karpiski, J. (2006) Fresh bone marrow and periosteum transplantation for cartilage defects of the knee. Transplantation Proceedings, 38, 318-319.

doi:10.1016/j.transproceed.2005.12.075

[20] Olivos-Meza, A., Fitzsimmons, J.S., Casper, M.E., Chen, Q., An, K.-N., Ruesink, T.J., O'Driscoll, S.W. and Reinholz, G.G. (2010) Pretreatment of periosteum with TGF$\beta 1$ in situ enhances the quality of osteochondral tissue regenerated from transplanted periosteal grafts in adult rabbits. Osteoarthritis Cartilage, 18, 1183-1191. doi:10.1016/j.joca.2010.06.003

[21] Mahmut, A., Mithat, Ö., Ahmet, G., Mehmet, H., Oğuz, T. and Özlem, C. (2010) The healing of full-thickness articular cartilage defects in rabbits: Successful results with fibroblast growth factor. Joint Diseases and Related Surgery, 21, 147-152.

[22] Ulutaş, K., Menderes, A., Karaca, C. and Ozkal, S. (2005) Repair of cartilage defects with periosteal grafts. The 
British Association of Plastic Surgeons, 58, 65-72. doi:10.1016/j.bjps.2004.05.010

[23] Depalma, A.F., Mckeever, C.D. and Subin, D.K. (1996)

Process of repair of articular cartilage demonstrated by histology and autoradiography with tritiated thymidine. Clinical Orthopaedics and Related Research, 48, 229242. 